\title{
Positive Effects of Massage Therapy on a Patient with Narcolepsy
}

\author{
Robyn Hill, RMT, Amanda Baskwill, BEd, RMT* \\ Massage Therapy Program, Humber Institute of Technology and Advanced Learning, Toronto, ON, Canada
}

Purpose: The purpose of this case report was to investigate the effects of massage therapy on the sleep patterns of a woman with narcolepsy.

Participant: The 23-year-old woman's primary symptoms included excessive daytime sleepiness and periodic leg movements (PLM), which were associated with her diagnoses of both narcolepsy and cataplexy.

Intervention: Five 45-minute massage therapy treatments were administered over a five-week period. The patient's sleep patterns were recorded each week before the treatment. A final measurement was recorded in the sixth week. The sleep patterns were monitored using the Leeds Sleep Evaluation Questionnaire, which included ten visual analogue scales.

Results: The results of this case report included an improvement in getting to sleep by $148 \%$, an improvement in quality of sleep by $1100 \%$, an improvement in awake following sleep by $121 \%$, and an improvement in behaviour following wakening by $28 \%$ using the Leeds Sleep Evaluation Questionnaire.

Conclusion: This case report suggests that massage therapy had a positive effect on this patient with narcolepsy. Further research is needed to investigate the effects of massage therapy on narcolepsy and sleep patterns.

KEY WORDS: narcolepsy; sleep patterns; massage therapy; getting to sleep; quality of sleep; awake following sleep; behaviour following wakening

\section{INTRODUCTION}

Narcolepsy is an excessive sleepiness disorder which is characterized by excessive daytime sleepiness, sleep paralysis, cataplexy, nocturnal sleeping patterns, and hypnagogic hallucinations. ${ }^{(1)}$ Some of these symptoms, such as sleep paralysis and hypnagogic hallucinations, are thought to be the result of dissociated REM sleep. ${ }^{(2)}$ Narcolepsy is often first experienced by a person in their 20 s or 30 s. $^{(3)}$ There is no cure for narcolepsy, but different medications can be used to offset symptoms experienced. ${ }^{(2,3)}$
Pharmacological treatment tries to stimulate the individual during the day and reduce sleep disturbances at night. Studies suggest that individuals with narcolepsy struggle with educational and occupational problems despite pharmacological treatment. ${ }^{(4)}$ It is believed that maintaining regular nocturnal sleep habits and attention to sleep hygiene can help to optimize daytime alertness. ${ }^{(5)}$

Massage therapy (MT) is a health care option that can aid in the prevention and treatment of pain and physical dysfunction through physical manipulation. ${ }^{(6)}$ Although massage therapy assessment and treatment often focus on physical outcomes, a wellrecognized review of MT research suggests that massage therapy has a significant impact on psychological outcomes, such as reduction in anxiety and depression. ${ }^{(7)}$ Some evidence suggests that massage therapy improves sleep in different populations. ${ }^{(8-14)}$ No studies have specifically been conducted on the effect of massage therapy on individuals with narcolepsy.

The effects of massage therapy on sleep have been studied in a number of different populations. One study investigated the effects of massage therapy and sleep in infants with low birth weight. ${ }^{(14)}$ This study showed that the group of infants with low birth rates that received the massage therapy intervention was more alert during the day. This study also showed that the massage therapy intervention facilitated positive sleep patterns in the infants, which included reducing breathing disorders such as sleep apnea and snoring, and increasing daytime alertness and improving the quality of sleep.

A study investigating the effects of massage therapy on elders with sleep disorders in a nursing home showed that the intervention group which received the massage therapy treatments effectively produced a relaxation response in the elderly individuals as measured by their resting heart rate, systolic and diastolic blood pressure, and scores on the visual analogue scale (VAS). ${ }^{(15)}$ In some of the cases, the intervention induced sleep, decreased sleep disruptions, and improved the overall sleep quality.

This case report is the first to investigate the research question, "Does massage therapy have an effect on a patient with narcolepsy?" It was hypothesized that massage therapy would improve the sleep 
patterns of a patient with narcolepsy. As the intervention would be conducted during the day, the treatment plan focused on providing a massage therapy intervention designed to be stimulating rather than sedative. Stimulating massage therapy techniques are thought to act as wake-promoting agents or stimulants to the body's sensory neurons. ${ }^{(16)}$ It was anticipated that this would promote proper sleep patterns by helping the participant stay alert during the day and possibly reduce sleep disturbances at night.

\section{METHODS}

This study is a prospective single-case design in which massage therapy was provided to a patient with narcolepsy. The intervention was provided once a week for 45 minutes over five weeks. The patient's sleep behaviours were recorded using the Leeds Sleep Evaluation Questionnaire. ${ }^{(17)}$

\section{Patient Profile}

The participant was a 23-year-old teacher who was diagnosed with narcolepsy with cataplexy five years ago. Her symptoms include excessive daytime sleepiness, periodic leg movements (PLM), and cataplexy. She experiences fatigue throughout the day which makes it more difficult to fall asleep at night. When she wakes up in the morning to go to work, she feels as though she has not had a restful sleep and continues to be tired throughout the day. When she comes home from work in the evenings, she feels exhausted and takes a nap, which sometimes turns into sleep for over 2-3 hours. This makes it harder for her to fall asleep later at night. She tries to take shorter, more frequent naps when possible, so that she does not fall asleep for long periods of time, but finds it difficult to do once she falls asleep. The participant describes her sleep patterns as frustrating and difficult to overcome.

\section{Massage Therapy Intervention}

The intervention in this study was massage therapy and was administered by a student massage therapist who provided massage techniques within the scope of practice in Ontario. At this point in her program, the student had completed about 1700 hours of her 2430-hour program. The massage therapy intervention was designed to positively impact the patient's sleep patterns by stimulating daytime wakefulness. Each treatment began with superficial reflex and superficial fluid techniques. ${ }^{(16)}$ The treatment primarily included rapid tapotement and petrissage applied to the anterior and posterior surfaces of legs, the back, and both arms. The protocol was pragmatic, meaning that the therapist could choose the exact techniques, the length of time each was administered, and the order. Each treatment was once a week for forty-five minutes, for five weeks. In the sixth week of the study, the questionnaire was completed with no intervention.

\section{Data Collection and Analysis}

The Leeds Sleep Evaluation Questionnaire (LSEQ) was used to measure sleep patterns. ${ }^{(17)}$ This tool uses ten visual analogue scales, each $100 \mathrm{~mm}$, in four areas. These areas include the concerning ease of getting to sleep (GTS), the perceived quality of sleep (QOS), the ease of awakening from sleep (AFS), and the integrity of early-morning behaviour following wakefulness (BFW). The questionnaire was completed in the week prior to the start of the massage therapy intervention and prior to the intervention in each of the following weeks. One final questionnaire was completed the week following the last massage therapy treatment. Each visual analog scale (VAS) was measured and the resulting score recorded for analysis. ${ }^{(18)}$

The data were organized by grouping the visual analog scales by section (ease of getting to sleep, the perceived quality of sleep, the ease of awakening from sleep, and the integrity of early-morning behaviour following wakefulness). As such, ease of getting to sleep included the results of three VASs, perceived quality of sleep included two VASs, ease of awakening from sleep included two VASs, and the integrity of early-morning behaviour following wakefulness included the results of three VASs. The data were analyzed by calculating the percent change using the results of the final data collection point, subtracting the results from the first data collection point, and dividing it by the results of the first data collection point and multiplying by 100 ([Data 6 - Data 1]/Data $1 \times 100)$. The total change, found by combining the results of all of the visual analog scales into a composite score and using the same formula as above, was also calculated.

\section{RESULTS}

Overall, there was a $123 \%$ total change on the Leeds Sleep Evaluation Questionnaire between the initial and final use of the tool. Higher numbers indicate improvement on each of the visual analog scales, so the change is an improvement in symptoms (Figure 1).

In the first area, getting to sleep, a $148 \%$ improvement was seen following the six-week period (Figure 2). For the questions about quality of sleep (QOS), there was $1100 \%$ improvement from the beginning to end of the study (Figure 3). The questions related to awake following sleep (AFS) showed a $121 \%$ change (Figure 4). Finally, behavior following wakening (BFW) improved by $28 \%$ (Figure 5 ). 


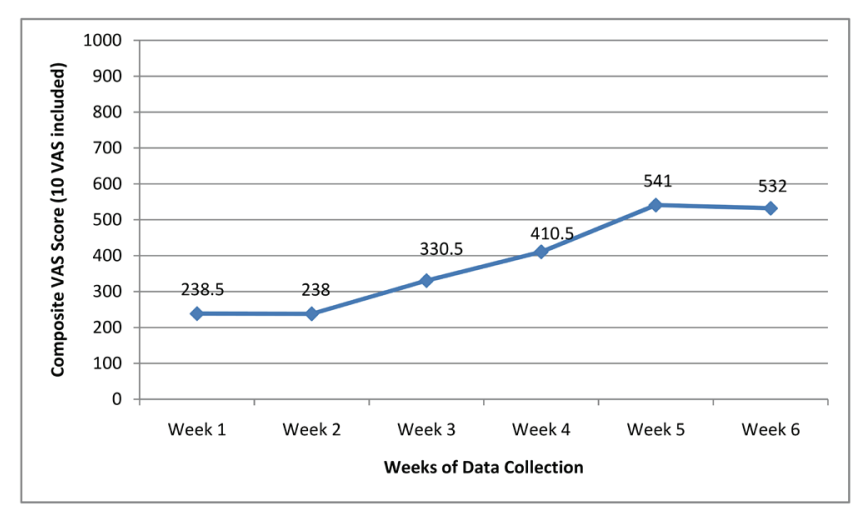

FIGURE 1. Global Leeds Sleep Evaluation Questionnaire Scores.

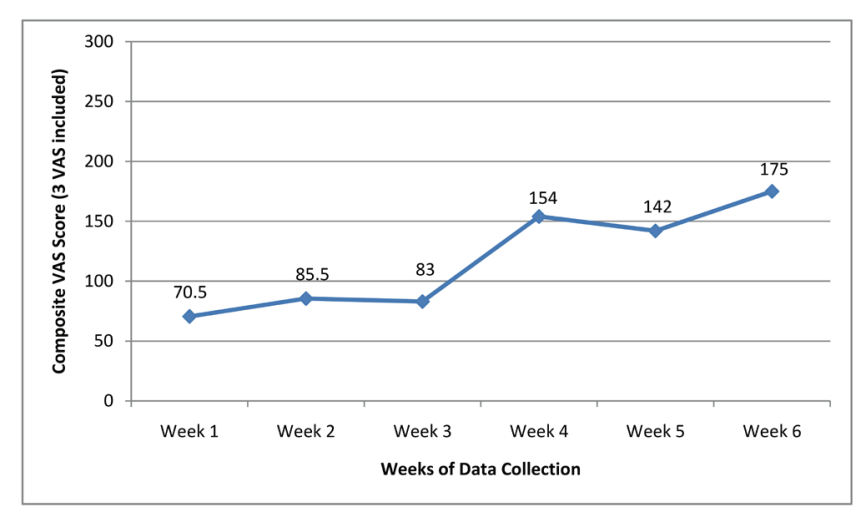

FIGURE 2. Getting to Sleep.

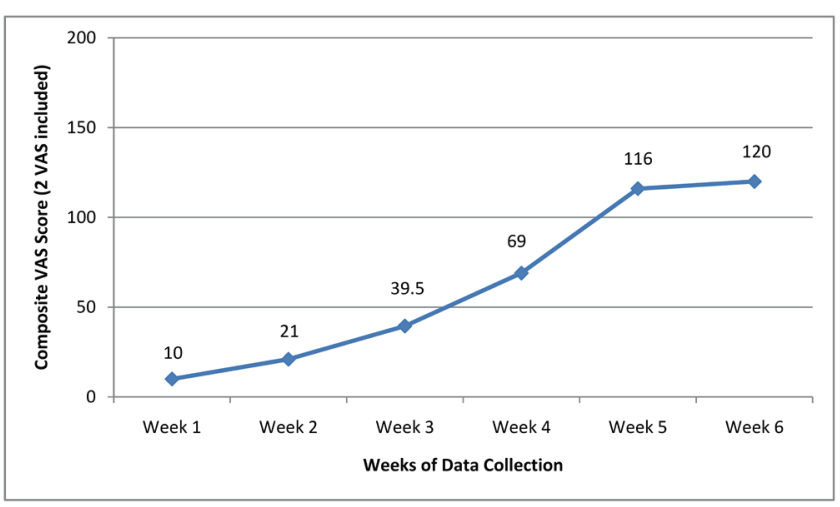

FIGURE 3. Quality of Sleep.

\section{DISCUSSION}

The results of this study suggest that massage therapy had a positive effect on the sleep patterns of this participant with narcolepsy. In particular, substantial improvement was experienced by the participant in the area of quality of sleep. From Figure 3, one can see that the data points suggest a steady improvement in quality of sleep over the study with the results tapering off in the last

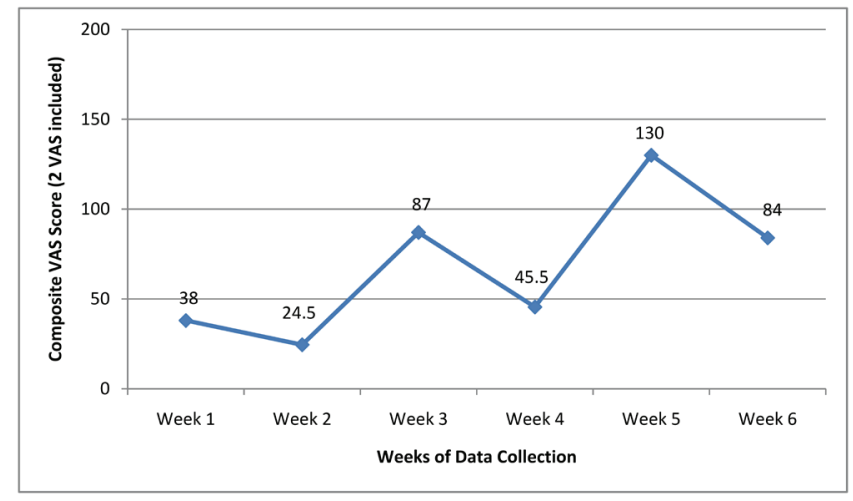

FIGURE 4. Awakening Following Sleep.

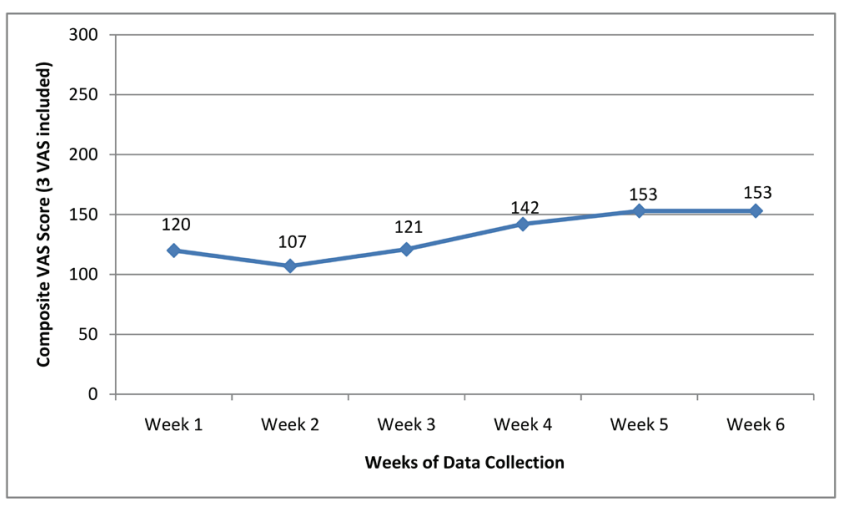

FIGURE 5. Behaviour Following Wakening.

week of the study. Future studies might consider a longer time period to see if the impact of weekly treatments on quality of sleep plateaus following four treatments.

The characteristic of getting to sleep also showed a fairly steady improvement over the study, although the percent change from beginning to end of the study was not as large. Awake following sleep shows an erratic pattern, and it is unclear whether the percent change seen was as a result of the massage therapy intervention or other factors. Behaviour following wakening did not show substantial improvement, and this would be interesting to explore further. It is worth considering whether this study was sufficiently long to capture any effect massage therapy might have on this characteristic.

The results of this study suggest that these massage therapy treatments improved the participant's sleep patterns, in particular by helping her get to sleep and improving her quality of sleep. A study regarding the effects of massage therapy and sleep ${ }^{(19)}$ found similar results, whereby massage therapy improved sleep by reducing sleep latency due to less disturbed sleep. While massage therapy seemed to benefit the participant in this study, these results may not generalize to all patients with narcolepsy. In addition, this study cannot suggest a cause and effect relationship 
between massage therapy and narcolepsy; a more rigorous study is needed.

It is recommended that future research focus on the population of individuals with narcolepsy and sleep behaviours in various populations. Based on the experience of this case study, the authors suggest the following considerations:

- Timing of the intervention: Does the timing of the intervention of massage therapy have an effect on the outcome? Timing the stimulating massage therapy treatment with times of fatigue may increase the effectiveness. Similarly, soothing massage therapy treatment closer to bedtime may also alter the impact of massage therapy on sleep behaviours.

- Dosage of the intervention: It is important to better understand the amount of massage therapy that is needed to create change in sleep patterns and to maintain that change. Using different lengths of treatment plans will help to better understand the appropriate dosage of massage therapy.

- Measurement tools: While the Leeds Sleep Evaluation tool was easy to administer and analyze, future studies may choose to use additional or alternate measurement tools. Due to the importance of REM sleep patterns to individuals with narcolepsy, it would be interesting to investigate whether or not massage therapy treatments have an impact by altering the REM patterns during sleep.

\section{CONCLUSION}

This single-case report was conducted to answer the question, "Does massage therapy have an effect on a patient with narcolepsy?' Results of this study suggest that massage therapy can improve sleep patterns in a patient with narcolepsy. In particular, quality of sleep and the ability to get to sleep showed the most promising impact. Further clinical research on massage therapy and narcolepsy is needed to better understand the preliminary results of this case.

\section{ACKNOWLEDGMENTS}

The authors wish to acknowledge Monica Golebiowski, Student Massage Therapist, who provided the massage therapy intervention in this study, and the participant of this study who generously volunteered her time to participate.

\section{CONFLICT OF INTEREST NOTIFICATION}

The authors declare there are no conflicts of interest.

\section{COPYRIGHT}

Published under the CreativeCommons AttributionNonCommercial-NoDerivs 3.0 License.

\section{REFERENCES}

1. Jara C, Popp R, Zulley J, Hajak G, Geisler P. Determinants of depressive symptoms in narcoleptic patients with and without cataplexy. J Nerv Mental Dis. 2011;199(5):329-334.

2. Dauvilliers Y, Arnulf I, Mignot E. Narcolepsy with cataplexy. The Lancet. 2007;369(9680):499-511.

3. Daniels E, King MA, Smith IE, Shneerson JM. Health-related quality of life in narcolepsy. J Sleep Res. 2001;10(1):75-81.

4. Overeem S, Mignot E, van Dijk JG, Lammers GJ. Narcolepsy: clinical features, new pathophysiologic insights and future perspectives. J Clin Neurophysiol. 2001;18(2):78-105.

5. Zeman A, Britton T, Douglas N, Hansen A, Hicks J, Howard $\mathrm{R}$, et al. Narcolepsy and excessive daytime sleepiness. BMJ. 2004;329(7468):724-728.

6. Government of Ontario. Massage Therapy Act, 1991. Ottawa, ON: Queen's Printer; 1991.

7. Moyer CA, Rounds J, Hannum JW. A meta-analysis of massage therapy research. Psychol Bull. 2004;130(1):3-18.

8. Oliveira D, Hachul H, Tufik S, Bittencourt L. Effect of massage in postmenopausal women with insomnia - a pilot study. Clinics. 2011;66(2):343-346.

9. Field T, Hernandez-Reif M, Diego M, Fraser M. Lower back pain and sleep disturbance are reduced following massage therapy. J Body Mov Ther. 2007;11(2):141-145.

10. Field T, Kilmer T, Hernandex-Reif M, Burman I. Preschool children's sleep and wake behavior: effects of massage therapy. Early Child Dev Care. 1996;120(1):39-44.

11. Field T, Diego M, Cullen C, Hernandez-Reif M, Sunshine W, Douglas S. Fibromyalgia pain and substance P decrease and sleep improves after massage therapy. J Clin Rheumatol. 2002;8(2):72-76.

12. Nerbass FB, Feltrim MIZ, de Souza SA, Ykeda DS, LorenziFilho G. Effects of massage therapy on sleep quality after coronary artery bypass graft surgery. Clinics. 2012;65(11): $1105-1110$.

13. Goldstein Ferber S, Laudon M, Kuint J, Weller A, Zisapel $\mathrm{N}$. Massage therapy by mothers enhances the adjustment of circadian rhythms to the nocturnal period in full-term infants. J Dev Behav Pediatr. 2002;23(6):410-415.

14. Kelmanson IA, Adulas EI. Massage therapy and sleep behaviors in infants born with low birth weight. Complement Ther Clin Pract. 2006;12(3):200-205.

15. Nelson R, Coyle C. Effects of a bedtime massage on relaxation in nursing home residents with sleeping disorders. Act Adapt Aging. 2010;34(3):216-231.

16. Andrade CK, Clifford P. Outcome-Based Massage: From Evidence to Practice. New York, NY: Wolters Kluwer/Lippincott Williams \& Wilkins; 2008.

17. Parrott AC, Hindmarch I. Factor analysis of a sleep questionnaire. Psych Med. 1978;8(2):325-329.

18. Leeds Sleep Evaluation Questionnaire (LSEQ). In: Shahid A, Wilkinson K, Marcu S, Shapiro CM. editors. STOP, THAT, and 
One Hundred Other Sleep Scales. New York, NY: Springer; 2012:211-213.

19. Ejindu A. The effects of foot or facial massage on sleep induction, blood pressure, pulse and respiratory rate: crossover pilot study. Complement Ther Clin Pract. 2007;13(4):266-275.
Corresponding author: Amanda Baskwill, BEd, RMT, Massage Therapy Program, Humber Institute of Technology and Advanced Learning, 205 Humber College Blvd., Toronto, ON M9W 5I7, Canada

E-mail: amanda.baskwill@humber.ca 